\title{
TRICOBEZOARES NO ESTÔMAGO, DUODENO E ÍLEO
}

\author{
TRICHOBEZOARS IN THE STOMACH, DUODENUM AND ILEUM
}

\author{
Fransber Rondinelle Araújo Rodrigues ${ }^{1}$ \\ Carlos Augusto Teixeira Cruz, TCBC-DF ${ }^{2}$ \\ André Luiz Vianna, TCBC-DF ${ }^{3}$
}

\section{INTRODUÇÃO}

O termo bezoar é usado para designar o acúmulo de substâncias ingeridas no estômago ou intestino do homem ou outros animais. ${ }^{1}$ São divididos em fitobezoares, formados por constituintes de alimentos que não são digeridos; tricobezoares, formados por pêlos; lactobezoares, decorrentes da ingestão de fórmulas lácteas altamente concentradas por neonatos; e uma quarta classe de bezoares, formados por substâncias variadas, como colas e medicamentos. ${ }^{2}$ São infreqüentes tanto as descrições de mais de um tricobezoar no mesmo indivíduo, quanto sua localização extragástrica ${ }^{1}$. O objetivo do presente estudo é relatar a presença de tricobezoares com localizações gástrica, duodenal e ileal em paciente tratado no serviço de Cirurgia Geral do Hospital Universitário de Brasília.

\section{RELATO DO CASO}

A.F.A., 21 anos, sexo feminino, apresentou cólicas abdominais eventuais associadas à ingestão de alimentos sólidos. Conviveu com esses sintomas durante um ano, quando passou a apresentar dor em cólica, inicialmente em fossa ilíaca e flanco esquerdos, tornando-se difusa, e vômitos de odor fétido. Evoluiu com distensão abdominal, quando procurou assistência médica. Ao exame físico, o abdômen estava distendido, com tumor em hipocôndrio esquerdo, epigastro e mesogastro, endurecido, bem delimitado, medindo $12 \times 8 \mathrm{~cm}$, indolor e móvel. O toque retal revelou tumor extrínseco comprimindo a parede anterior do reto, de consistência fibroelástica, doloroso à mobilização. A radiografia de abdômen mostrou distensão de alças de intestino delgado em hipocôndrio direito, com níveis hidroaéreos. A endoscopia digestiva alta diagnosticou tricobezoar. A laparotomia evidenciou corpo estranho intragástrico, em fundo e corpo, moldando a luz do órgão, com $23 \mathrm{~cm}$ x $10 \mathrm{~cm}$; o duodeno apresentava-se dilatado e com corpo estranho de $12 \mathrm{~cm}$ x $6 \mathrm{~cm}$; no íleo terminal observou-se outro corpo estranho de $12 \mathrm{~cm} \mathrm{x} 4 \mathrm{~cm}$, que obstruía a luz do órgão e causava dilatação de alças intestinais a montante. Realizou-se gastrotomia anterior longitudinal de $10 \mathrm{~cm}$, por onde se retirou o tricobezoar gástrico (Figura 1) e o duodenal. Não foi possível a ordenha do tricobezoar ileal para o cólon, visto que encontrava-se fixado à parede intestinal. Foi então retirado por ileotomia longitudinal (Figura 2).

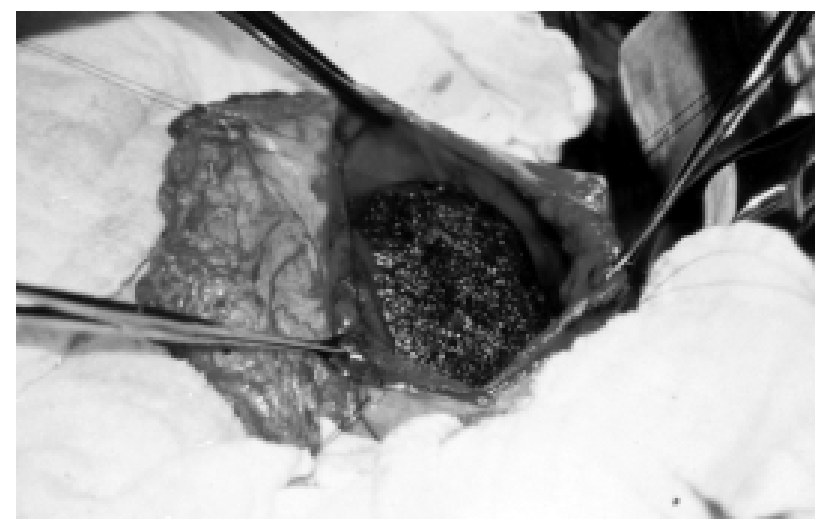

Figura 1 - Gastrotomia anterior evidenciando tricobezoar gástrico.

1. Acadêmico do Curso de Medicina da Universidade de Brasília.

2. Professor Adjunto do Departamento de Clínica Cirúrgica da Faculdade de Ciências da Saúde da Universidade de Brasília.

3. Professor Adjunto do Departamento de Clínica Cirúrgica da Faculdade de Ciências da Saúde da Universidade de Brasília.

Recebido em 19/5/99

Aceito para publicação em 10/12/99

Trabalho realizado no Departamento de Clínica Cirúrgica da Faculdade de Ciências da Saúde da Universidade de Brasília. Hospital Universitário de Brasília, Brasília, DF. 


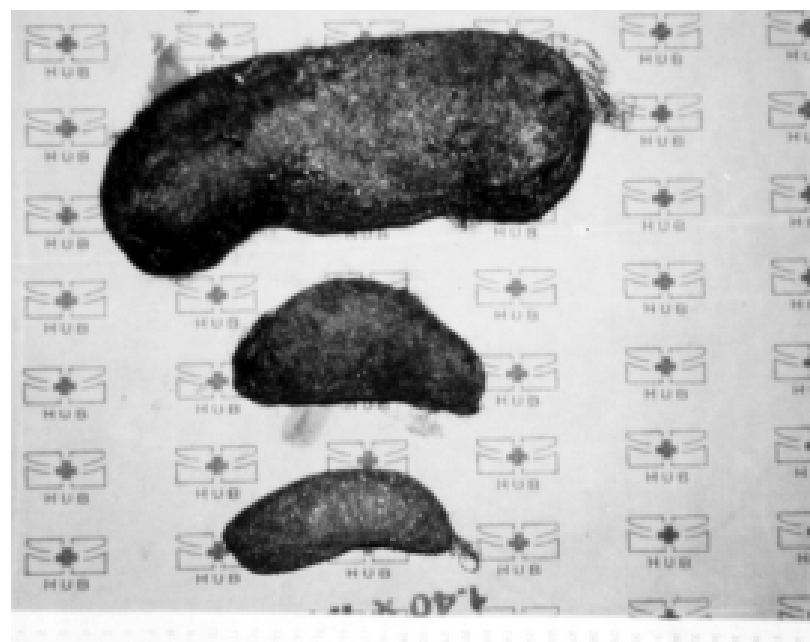

Figura 2 - Tricobezoares gástrico, duodenal e ileal, respectivamente.

A paciente evoluiu bem até o terceiro dia pós-operatório, quando apresentou sinais de irritação peritoneal, sendo reoperada com a hipótese diagnóstica de deiscência da gastro ou enterorrafia. $\mathrm{O}$ achado operatório revelou deiscência de sutura ileal e procedeu-se à ressecção do segmento envolvido, com anastomose término-terminal. A paciente evoluiu bem e recebeu alta no sétimo dia após a segunda operação.

\section{DISCUSSÃO}

Segundo DeBakey, 91,4\% dos casos de tricobezoar ocorrem em mulheres, com maior incidência entre os 10 e 19 anos. ${ }^{1}$ As queixas mais freqüentes são dor abdominal, náuseas e vômitos, seguidos de sensação de plenitude e desconforto epigástrico, distensão abdominal, anorexia, perda de peso e alteração do ritmo intestinal. ${ }^{1,2}$ No exame físico, o achado mais comum é de tumor epigástrico móvel e geralmente não doloroso. Estes sintomas e sinais foram observados no presente caso. Halito- se, alopécia, palidez, emagrecimento e crepitações sobre o tumor estão freqüentemente presentes. ${ }^{1,2} \mathrm{O}$ toque retal pode revelar um tumor extrínseco, ${ }^{2}$ o que foi relevante no presente caso, pois sugeriu a presença de bezoar de localização intestinal, após a confirmação endoscópica de bezoar no estômago.

O quadro clínico de tricobezoar é inespecífico, o que dificulta o diagnóstico clínico e exige alto grau de suspeição. ${ }^{1,2}$ A forma de apresentação clínica no presente estudo pode ser considerada típica, porém, o achado cirúrgico de tricobezoares com localização gástrica, duodenal e ileal não é freqüente. ${ }^{1}$

DeBakey observou que apenas $9 \%$ dos pacientes com tricobezoar apresentavam algum distúrbio psiquiátrico evidente. ${ }^{1}$ Contudo, ele suspeitou que a tricofagia, por si, pode representar um problema neurológico ou psiquiátrico. Outros autores postularam que a tricofagia é reflexo de tensão emocional, advinda principalmente de conflitos familiares. ${ }^{4}$ Mais recentemente, este hábito foi inserido no contexto dos transtornos obsessivo-compulsivos. ${ }^{5}$ No caso relatado, não se observou tais alterações. Além disso, a paciente negava ingestão de cabelos, comportamento observado em $50 \%$ dos pacientes relatados por DeBakey. ${ }^{1}$

Obstrução intestinal, como observado neste caso, possivelmente decorre do desprendimento de uma parte do bezoar gástrico original, que pode se alojar em qualquer local do intestino delgado e grosso. ${ }^{1}$ Outras complicações podem ocorrer, como ulcerações, perfuração, hemorragias, fístulas e intussuscepção. ${ }^{2,3}$.

Desde o início do século, o tratamento de tricobezoares é cirúrgico. ${ }^{1}$ Tentativas de tratamento endoscópico foram feitas, entretanto, este método só se provou eficaz para fitobezoares. ${ }^{4,5}$ Além da dificuldade de se fragmentar os tricobezoares gástricos por gastroscopia, há o perigo da liberação de porções menores do bezoar, que podem causar complicações, como obstrução intestinal. 2,3

Uma vez desencadeadas as complicações de um tricobezar, como no caso relatado, a morbimortalidade aumenta consideravelmente. ${ }^{1}$ Sendo assim, o paciente deve ser operado assim que o diagnóstico seja confirmado.

\begin{abstract}
A 21-year-old girl presents with a 15-day history of epigastric pain and vomiting. Ten days later, constipation and abdominal distension followed. The patient reported similar epigastric pain and vomiting for 6 days last year. A painless epigastric 12-by-8-centimeter tumor was observed on abdominal palpation, and rectal examination revealed an extrinsic tumor compressing anterior rectal wall. Upper digestive endoscopy showed a trichobezoar. Upon laparotomy, one gastric and one duodenal trichobezoar were removed through an anterior gastrotomy. Another one was found 40 centimeters proximal to the ileo-cecal valve and removed through a proximal ileotomy. Trichobezoar is an unspecific disease, which diagnosis is made upon suspicion. Early recognition of bezoars is important because morbidity and mortality rise considerably once complications occur. We illustrate this case to emphasize the need for early recognition and surgical management in order to reduce morbimortality.
\end{abstract}

Key Word: Trichobezoar. 


\section{REFERÊNCIAS}

1. DeBakey M, Ochsner A-Bezoars and concretions: A comprehensive review of the literature with na analysis of 303 cases and a presentation of 8 aditional cases. Surgery 1938; 4:934-963; Surgery 1939; 5:132-160.

2. Lamerton AJ - Trichobezoar: Two case reports - A new physical sign. Am J Gastroenterol 1984; 79:354-356.

3. Cardoso SC, Santos VR - Perfuração gástrica por tricobezoar. Rev Col Bras Cir 1998; 15(6): 430-431.

4. Dietrich NA, Gau FC - Postgastrectomy phytobezoars: endoscopic diagnosis and treatment. Arch Surg 1985; 120:432-435.
5. Kuo JY, Mo LR, Tsai CC, et al. - Endoscopic fragmentaion of gastric phytobezoar by eletrohydraulic lithotripsy. Gastrointestinal Endoscopy 1993; 39(5): 706-708.

\section{ENDEREÇO PARA CORRESPONDÊNCIA}

Dr. André Luiz Vianna

Fac. Ciências da Saúde

Depto. Clínica Cirúrgica

Campus Universitário Darcy Ribeiro - Asa Norte

70919-970 - Brasília-DF 